\title{
A criterion for rotational augmentation based on a boundary-layer analysis
}

\author{
W.-G. Früh ${ }^{1}$ and A.C.W. Creech ${ }^{2}$ \\ ${ }^{1}$ Institute of Mechanical, Process and Energy Engineering, School of Engineering and Physical Sciences \\ Heriot-Watt University \\ Riccarton - Edinburgh EH14 4AS (UK) \\ Phone number: +44 131451 4374, e-mail: w.g.fruh@hw.ac.uk \\ ${ }^{2}$ Institute of Energy Systems, School of Engineering, University of Edinburgh \\ Kings Buildings, Edinburgh EH9 3JL (UK), e-mail: a.creech@ed.ac.uk
}

\begin{abstract}
Rotational augmentation is a well-known phenomenon of rotating aerofoils on wind turbines and helicopters where the lift or torque is improved compared to that of stationary aerofoils.
\end{abstract}

We present an analysis which is based on the concept of a developing boundary layer and the Ekman boundary layer using a 3D Finite-Element Analysis of the developing laminar boundary layer over a flat plate in a rotating frame of reference. A parametric analysis addresses the Reynolds number, the Ekman number and the speed ratio in turn.

The results demonstrate that the surface's rotation limits the boundary layer to the thinner of the two boundary layers, and that it induces a span-wise flow in the radial direction which in turn leads to Ekman suction. This Ekman suction provides a mechanism to counteract an adverse pressure gradient and thus prevent stall. The criterion derived from this was successfully applied and tested against observations and full CFD results.

\section{Key words}

Wind turbine aerodynamics, Rotational augmentation, Boundary layer analysis

\section{Introduction}

When analysing the performance of wind turbines using the concepts of the lift and drag forces from the aerofoilshaped rotor blades, the actual performance may, under some conditions, be better than expected. Earlier work for helicopter rotors $[1,2]$ and then extended to wind turbines [3], succeeded in associating the enhanced performance with suppressing stall. In fixed-wing aerofoils, the lift coefficient increases with angle of attack up to a maximum, beyond which the lift drops substantially off and the drag increases rapidly. This phenomenon, called 'stall', is associated with the separation of the boundary layer from the aerofoil surface. As a result, a wake forms which leads to the reduced lift and enhanced drag. For rotating blades, it was found that the onset of stall is delayed to higher angles of attack compared to that of stationary, but otherwise identical, aerofoils. For that reason, the phenomenon has been termed 'rotational augmentation'. Besides the result of improved lift and extended range of attached flow, the main characteristic in the velocity fields was a noticeable cross flow within the boundary layer radially outwards towards the tip of the blade. While a number of models have been proposed to incorporate 3D effects included rotational augmentation, e.g. [4], there is no clear basis for formulating a succinct necessary or sufficient criterion for occurrence of rotational augmentation.

The practical aim of this paper is to suggest a criterion for rotational augmentation based on the boundary dynamics due to the combination of the developing boundary layer of the flow over the aerofoil on one hand with the Ekman boundary layer from the balance of Coriolis and viscous terms on the other hand. To identify the most basic underlying processes, we will first review the flow features associated with this phenomenon in $\S 1$.A and then introduce the forces used to explain the flow features in $\S 1$.B before proposing an explanation through boundary layer theory.

\section{A. Flow features associated with rotational augmentation}

Detailed pressure maps from tests with the NREL turbine and high-resolution CFD simulations, demonstrated that augmented lift occurred when regions existed on the blade where the boundary layer had separated from the surface but then became re-attached to the surface $[3,5]$. 
Associated with re-attachment was also a radial pressure gradient and flow radially outward within the boundary layer. The interpretation by Schreck et al. centred on the role of the tip speed ratio as a key parameter. As the scaling in $\S 2$.A will show, this implies a hypothesis that the centrifugal terms in the equations of motions are in some way determining the process of re-attachment of the boundary layer. An earlier investigation by Dwyer and McCroskey was largely based on numerical analysis of laminar flow over a rotating flat plate and a symmetric aerofoil in order to identify the key fundamental aspects of the flow [2]. One of their key findings was that the flow can be expressed in terms of a Blasius-type boundary-layer coordinate, which suggests that the developing Blasius boundary layer is one of the key ingredients. Another was that the Coriolis force appeared to be more important than the centrifugal force for slender blades. Combining the indication of the importance of boundary layer dynamics and of the Coriolis term from these two findings suggests that another key ingredient might be the boundary layer resulting from the balance of viscous to Coriolis forces, the Ekman layer. Thirdly, Dwyer and McCroskey reported that the 'effects of rotation can be important and beneficial with regard to separation of the primary flow in regions of adverse pressure gradients' [2]. This observation provides the link between the observed flow structures with the performance improvement through rotational augmentation.

In summary, rotational augmentation is associated with span-wise flow, span-wise pressure gradients and reattachment of separated flow which can lead to rotationally augmented lift in three ways; enhanced pressure difference from modified attached flow, delay of flow separation, or re-attachment of separated flow.

\section{B. Physical Processes}

An order-of-magnitude analysis has shown that the Coriolis acceleration can reduce adverse pressure gradients and, with it, the onset of stall [6]. A boundary layer analysis over a rotating blade assumed some velocity profiles of laminar or turbulent boundary layers and a streaming angle for the cross flow [7,8]. That analysis identified the speed ratio, $\lambda_{r}=r \Omega / U_{0}$, as a key parameter besides the radius-to-chord ratio and a Reynolds number. While they also mention a Rossby number they did not identify the Ekman number and the resulting Ekman layer as a potential agent in the process. As a result, they had to assume a cross-flow profile, which they assumed to be identical for laminar and turbulent flow. Noting that the centrifugal and Coriolis terms play different roles, it was suggested that the centrifugal term would somehow cause the cross-flow while the Coriolis term would accelerate the flow in the chord-wise direction and thus overcome the adverse pressure gradient [8]. A possible contribution from the Ekman layer is hinted at in another boundarylayer analysis, which showed that the growth of the laminar boundary layer developing over a flat plate is limited so that its displacement thickness would level out at the displacement thickness of the Ekman layer [9].

\section{Methodology}

Here we aim to isolate the fundamental processes arising from the centrifugal and Coriolis terms, respectively, and thus contribute to the understanding of the mechanisms leading to boundary layer topologies and flow characteristics associated with rotational augmentation. Building on this understanding, a second practical aim is to propose a criterion for the presence of rotational augmentation. To separate the various factors, we have used the same approach as Dwyer and McCroskey [2] by reducing the problem to the simplest possible case. This means that we based our analysis around steady laminar flow over a rotating flat plate. To separate all possible terms, we use a set of three non-dimensional parameters, which include the Ekman number in addition to the (tip) speed ratio and the Reynolds number, and investigate the response of the flow to systematic variation in each parameter.

The resulting flow is intrinsically three-dimensional with the vertical variation of the mean flow, the boundary layer developing downstream from the leading edge, and the induced cross-flow. For that reason, the analysis discussed here is based on a 3D Finite-Element model of laminar flow over a flat plate in a rotating frame of reference, where the rotational axis is perpendicular to the plate's surface. This model was used for a series of parametric studies for the main three non-dimensional parameters, namely the Reynolds number, the Ekman number, and the speed ratio. These parameters and the basic theory are summarised in the following sub-section.

\section{A. Boundary layers}

The basic momentum equation for an incompressible fluid in a rotating frame of reference is

$$
\frac{\partial \mathbf{u}}{\partial t}+(\mathbf{u} \cdot \nabla) \mathbf{u}+2 \mathbf{\Omega} \times \mathbf{u}=-\frac{1}{\rho} \nabla p-|\Omega|^{2} \mathbf{r}_{p}+v \Delta \mathbf{u}
$$

Where $\mathbf{u}$ is the velocity vector, $\boldsymbol{\Omega}$ the vector of rotation of the system, $\rho$ the fluid density, $p$ the pressure, $\mathbf{r}_{\mathbf{p}}$ the distance from the axis of rotation, and $v$ the fluid's kinematic viscosity, e.g. [10].

In the absence of rotation, the balance of inertial to viscous terms is quantified by the Reynolds number when typical scales $U_{0}$ and $L$ are used to scale the velocities and length scales, respectively:

$$
\frac{(\mathbf{u} \cdot \nabla) \mathbf{u}}{v \Delta \mathbf{u}} \rightarrow \frac{U_{0}^{2} / L}{v U_{0} / L^{2}} \rightarrow R e=\frac{U_{0} L}{v}
$$

The standard developing laminar boundary layer over a flat plate is the Blasius layer with a thickness $\delta_{R e}$ given by

$$
\frac{\delta_{\operatorname{Re}}}{x}=\frac{5}{\sqrt{\operatorname{Re}_{x}}}
$$


where $\mathrm{x}$ is the stream-wise distance from the leading edge and $R e_{x}=U_{0} x / v$ the local Reynolds number using that distance as the length scale. Other standard measures of the boundary layer are the displacement thickness, $\delta^{*}$, and momentum thickness, $\theta$, respectively, e.g. [11]:

$$
\delta^{*}=\int_{0}^{\infty}\left(1-\frac{u}{U_{\infty}}\right) d z
$$

and

$$
\theta=\int_{0}^{\infty} \frac{u}{U_{\infty}}\left(1-\frac{u}{U_{\infty}}\right) d z
$$

In a rapidly rotating fluid, the Coriolis term becomes a major term in the force balance, and the momentum equations can be scaled by that term, so that the ratio of the viscous term to the Coriolis term is expressed as the Ekman number and defined as

$$
\frac{v \Delta \mathbf{u}}{2 \Omega \times \mathbf{u}} \rightarrow \frac{v U_{0} / L^{2}}{2 \Omega U_{0}} \rightarrow E=\frac{v}{\Omega L^{2}}
$$

The relative magnitude of the centrifugal and Coriolis terms are given by

$$
\frac{\Omega^{2} \mathbf{r}_{p}}{2 \boldsymbol{\Omega} \times \mathbf{u}} \rightarrow \frac{\Omega^{2} r}{2 \Omega U_{0}} \rightarrow \lambda_{r}=\frac{\Omega r}{U_{0}}
$$

This non-dimensional parameter has the same form as the tip speed ratio, well known for wind turbines, but uses the local radial position along the blade instead of the full length of the blade. Hence it is here referred to as the speed ratio.

On a boundary, which is perpendicular to the axis of rotation, the presence of the Coriolis terms leads to an Ekman boundary layer of thickness [10]

$$
\frac{\delta_{E}}{L}=\sqrt{E} \quad \text { or } \quad \delta_{E}=\sqrt{\frac{v}{\Omega}}
$$

with a velocity profile of

$$
\begin{aligned}
& u_{E}=U_{0}\left(1-e^{-z / \delta_{E}} \cos \left(\frac{z}{\delta_{E}}\right)\right) \\
& v_{E}=U_{0} \quad e^{-z / \delta_{E}} \sin \left(\frac{z}{\delta_{E}}\right)
\end{aligned}
$$

The Blasius profile and the two components of the flow in the basic Ekman layer are shown in Figure 1, where the vertical axis has been scaled against the Ekman layer thickness. As can be seen, the streamwise in velocity in the Ekman layer reaches a maximum larger than the freestream velocity at a distance from the wall of $z / \delta_{E}=3 \pi / 4$, while the cross-flow reaches a maximum at $z / \delta_{E}=\pi / 4$. Figure 1 also shows a standard Blasius layer with a thickness of $\delta=3 \pi / 4 / \delta_{E}$ to show the velocity profiles of these two types of boundary layer if they have a similar dept.

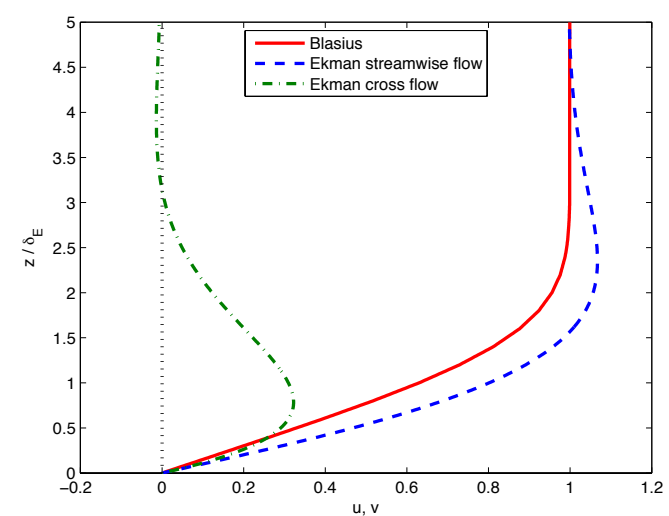

Fig. 1 Velocity profiles for the Blasius layer (solid line), and the Ekman layer with the dashed line for the streamwise velocity and the dash-dotted line for the cross flow velocity.

Using the streamwise velocity, eq.(9), in the definition of the displacement thickness, eq.(4), and integrating leads to a displacement thickness for the Ekman layer of

$$
\delta_{E}^{*}=\frac{1}{2} \sqrt{\frac{v}{\Omega}}
$$

Using it in the definition of the momentum thickness, eq.(5), and integrating leads to a momentum thickness for the Ekman layer of

$$
\theta_{E}=\frac{1}{8} \sqrt{\frac{v}{\Omega}}
$$

Fig. 2 shows that the displacement thickness is in the range of 0.1 to $1 \mathrm{~mm}$ for the typical range of rotation rates of large turbines. Fig. 1 showed substantial cross flow in the Ekman layer at a height of around $\delta_{E}$ and that the flow overshoots slightly at around $2 \delta_{E}$ before reaching the freestream velocity. To maintain mass continuity, a consequence of this Ekman layer is that a vertical velocity is induced at the top of the boundary layer, known as Ekman pumping of

$$
w_{E}=\frac{1}{2} E^{1 / 2} \zeta_{0}
$$

where $\zeta_{0}=\left(\partial v_{0} / \partial x-\partial u_{0} / \partial y\right)$ is the vorticity of the flow at the top of the Ekman layer. In the case of a blade rotating in the plane perpendicular to the incoming wind, this becomes $w_{E}=\sqrt{E} \frac{\lambda \omega}{\sqrt{1+\lambda^{2}}}$.

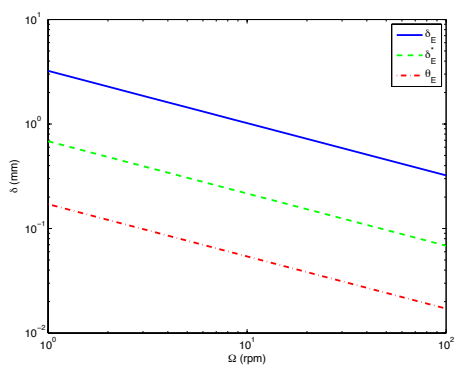

Fig. 2. Change of Ekman layer thicknesses against rotation rate. 


\section{B. Model configuration}

To investigate this developing boundary layer, a 3D FiniteElement code using COMSOL was set up to model the development of laminar flow over a flat plate section under the influence of Coriolis and centrifugal effects. The equation were scaled such that the plate was a square with unit area which formed the lower boundary in the central part of a domain of length 3 , width 1 , and height 1 , as shown in Fig. 3. The fluid density was set to unity and the viscosity equal to 0.1 . This resulted in free parameters directly proportional to the Reynolds number, equivalent to changing the inflow velocity, the Ekman layer, equivalent to changing the rotation rate, and the speed ratio, equivalent to choosing different sections along the blade.

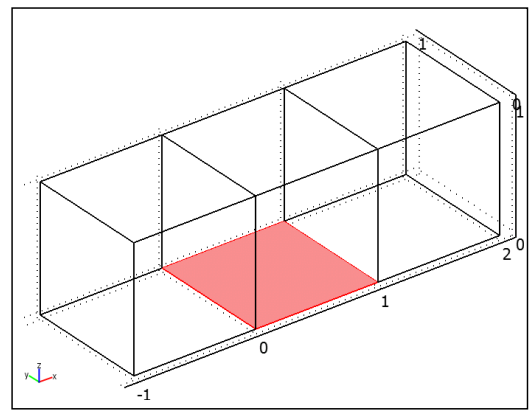

Fig. 3. The domain of the model with the solid surface indicated by the shaded area.

The first cuboid had a face with inlet velocity conditions of $u=U_{0}, v=w=0$ and a stress-free lower boundary. The central subdomain had no-slip condition at the lower boundary. The last subdomain had again a stress-free lower boundary as well as outflow conditions $(p=0)$ at the back. All the other boundaries were stress-free boundaries. While this was not the only possible choice of boundary condition, it gave the most stable results, and inspection of the solutions showed that the choice of side and top boundaries only affected the results within a distance of less than 0.1 from those boundaries.

The rotational effects, specified as body forces given by the Coriolis and centrifugal terms, were restricted to the volume above the central plate, such that the rotation vector was aligned with the vertical $z$-axis.

To resolve the boundary layer appropriately, the mesh resolution was highest at the lower boundary of the middle section. A range of element sizes were used to test the sensitivity of the results to model resolution. These ranged from a coarse mesh with element length of 0.1 to the finest possible mesh given the hardware limitations of 0.015 . For all of these, the results did not change for Ekman numbers as low as 0.003 .

Within the available grid resolution, the following ranges of parameters could be reliably explored:

- $\quad$ Reynolds number from 50 to 400

- $\quad$ Speed ratio from 1 to 20

- $\quad$ Ekman number down to 0.003
To minimise the effects of the side boundaries, the analysis centres around vertical profiles of the velocities in the centre of the surface, at $x=0.5$ and $y=0.5$. The three standard measures of the boundary layer thickness were calculated, the standard thickness as the height of the velocity maximum and the displacement and momentum thicknesses as defined in eq.s (4) and (5).

\section{Results}

A typical model result is shown in Fig. 4 as a vertical cross-section of the velocity magnitude and a set of streamlines starting at the inlet in a plane just above the lower boundary.

Key features are that the boundary layer (as indicated by the yellow and blue colours) initially develops like a standard laminar boundary layer but that the growth is terminated before the end of the surface. At the same time, the streamlines are deflected to the left as soon as they are above the rotating plate. In the following sections, first the boundary layer thickness will be presented, and then an application of the results to a typical wind turbine rotor blade.

\section{A. Boundary layer thickness}

Figure 5 shows the streamwise (a,c,e) and cross-flow $(b, d, f)$ velocity profiles for different values of each nondimensional parameter, the Reynolds number $(a, b)$, the Ekman number (c,d), and the speed ratio $(e, f)$. The stream wise velocity profiles (a) demonstrate the expected behaviour that the boundary layer thickness decreases as the Reynolds increases, which is accompanied by a decrease in the crossflow velocity (b). The change of the free-stream streamwise velocity with the boundary layer thickness is consistent with the conservation of mass satisfied in the numerical model.

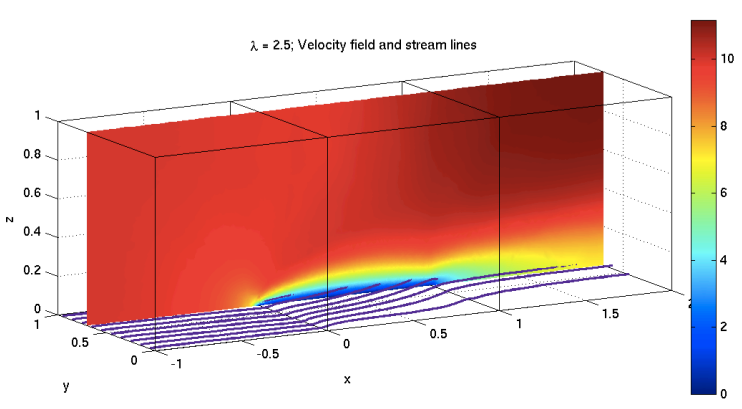

Fig. 4. A typical solution at $\Omega=5, R e=100, E=0.02$, and $\lambda_{r}=2.5$. Shown are a vertical slice of velcoty magnitude, and a set of streamlines originating at the inlet at a height of $z=0.02$. 
a) $R e$

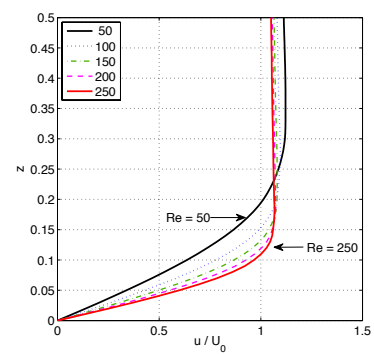

c) $E$

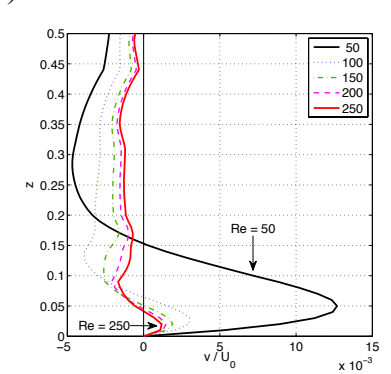

d)
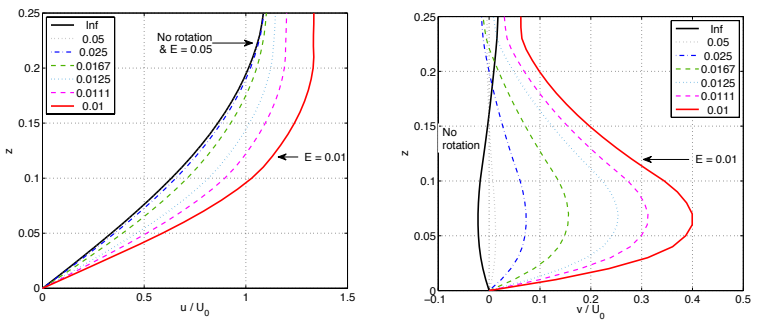

e) $\lambda_{r}$

f)
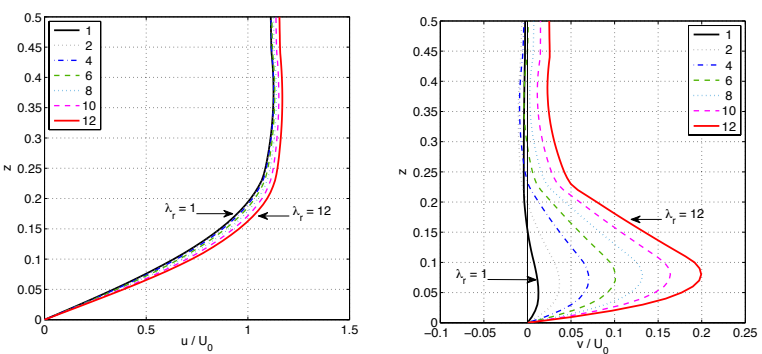

Fig. 5. Vertical profiles in the centre of the flat plate of the streamwise velocity in $(\mathrm{a}, \mathrm{c}, \mathrm{e})$ and of the cross-stream velocity in $(b, d, f)$, where the parameter for the different lines is the Reynolds number in $(a, b)$, Ekman number in $(c, d)$, and speed ratio in $(e, f)$.

The Ekman number also affects the streamwise profile (c) in which the thickness reduces as the Coriolis terms increases and the Ekman number decreases. In contrast to the Reynolds number effect, the cross flow velocities in the Ekman spiral (c) are enhanced as the Ekman layer becomes thinner. The reverse cross flow at the top of the boundary layer is consistent with [2]. The cross flow velocities are also enhanced by an increasing speed ratio (f) but the boundary layer thickness is not significantly affected by the centrifugal term.

The variation of the boundary layer thickness against the Reynolds and Ekman numbers is summarised in Fig. 6, which shows the displacement thickness for two sets of simulations where the Ekman number was changed, one at $R e=100$ (blue circles) and the other at $R e=400$ (red stars). Overlaid on the model results are two horizontal lines for the displacement thickness from eq. (4) for the two Reynolds numbers, and the inclined line for the Ekman layer from eq. (10). Comparing the numerical results with the analytical Blasius and Ekman layers suggests that the boundary layer develops like the Blasius layer for large Ekman numbers and like an Ekman layer at small Ekman numbers. The scaling of the observed boundary layer structure is ultimately determined by the smaller of the Ekman and Blasius displacement thicknesses.

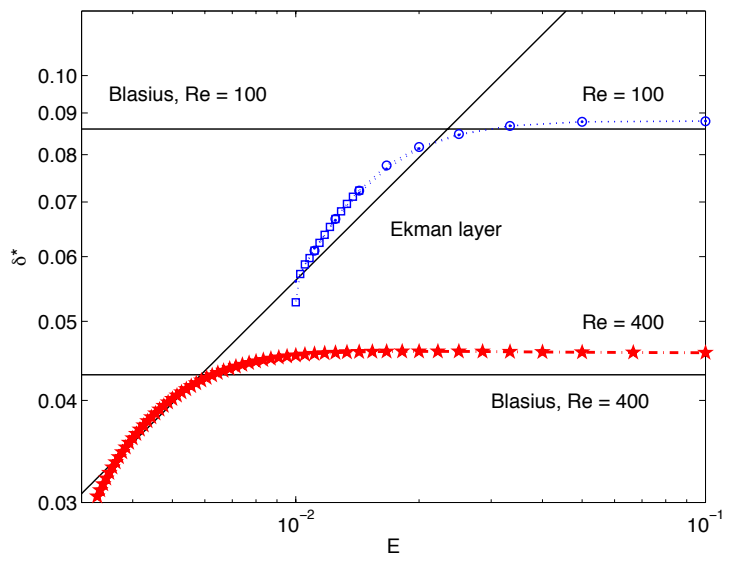

Fig. 6. The displacement boundary layer thickness against Ekman number.for two choices of Reynolds number, at $\lambda_{r}=1$.

As the Ekman layer thickness does not depend on the distance from the leading edge but the Blasius layer does, we can describe the developing boundary layer over the rotating surface such that it develops initially as Blasius layer until its displacement thickness reaches that of the Ekman layer. After that, the boundary layer does not develop further but remains at the Ekman layer thickness. One further implication is that a downward velocity at the top of the boundary layer is induced in the form of the Ekman suction given in eq. (12). This downward velocity of the air above the boundary layer provides a mechanism to keep the boundary layer attached to the surface or even to re-attach a previously separated boundary layer.

\section{B. Criterion for rotational augmentation}

As all the features of the flow previously associated with rotational augmentation are accounted for by the developing boundary layer over a rotating surface, we attempt here to formulate a criterion for rotational augmentation and apply it to the blade for which the rotational augmentation has been analysed in detail $[3,5]$.

Based on Figure 6, the criterion can be stated as:

\section{Rotational augmentation may occur}

$$
\text { if and only if } \delta_{E}{ }^{*}<\delta^{*}
$$

However, as the boundary layer over wind turbine blades develops to Reynolds numbers of $10^{6}$ to $10^{7}$, we have to apply that criterion to turbulent boundary layers. There is evidence that the fundamental flow structures were not altered for the turbulent boundary layer [2], and that rotation actually stabilised the developing boundary layer [12]. Also, the key features of the Ekman layer persist for the turbulent Ekman layer [13,14]. For that reason, we may transfer the criterion to the turbulent boundary layer by using the corresponding displacement thickness of the turbulent boundary layer compared against the unchanged Ekman layer thickness.

One standard description of the developing turbulent boundary layer is based on a time-averaged normalised 
velocity profile as $\mathrm{u}(\mathrm{z})=(z / \delta)^{1 / 7} \quad U_{0}$. Using this approximation, it is possible to quantify the displacement thickness of this turbulent boundary layer as

$$
\frac{\delta_{T}^{*}}{x}=\frac{0.0456}{R e_{x}^{1 / 5}}
$$

\section{Test of the criterion}

Here, the criterion is applied to the NREL turbine $[3,4,5]$ and to a large turbine like the Siemens SWT-2.3-93 at its cut-in and rated wind speeds. Figure 7.(a) shows a surface map of the criterion on the turbine blade of the NREL turbine $[3,5]$ at a wind speed of $10 \mathrm{~m} / \mathrm{s}$. The yellow area is where the developing boundary layer is thinner than the Ekman layer and the blue area is where the boundary layer is limited by the Ekman layer. Hence the blue areas indicated the extent to which rotational augmentation might be active. Figure 7.(b) and (c) show the corresponding results for the $2.3 \mathrm{MW}$ variable-speed turbine with a tip speed ratio of 7 at its cut-in wind speed of $4 \mathrm{~m} / \mathrm{s}$ and at its rated speed of $13 \mathrm{~m} / \mathrm{s}$, respectively. All three cases have some regions where rotational augmentation would be possible. For the NREL turbine, it is restricted to a fairly small region of the inbord part of the blade. While the extent with only $3 \%$ of the blade's surface is somewhat smaller than suggested by $[3,5]$, the location of the region agrees well with the observations by Schreck and Robinson [3]. The larger turbine, operating with a much higher tip speed ratio, has a larger area where rotational augmentation could occur, starting with $9 \%$ at the cut-in wind speed and rising to $20 \%$ at the rated wind speed. Increasing the wind speed further has little effect on the criterion as the rotation rate remains constant.

a)

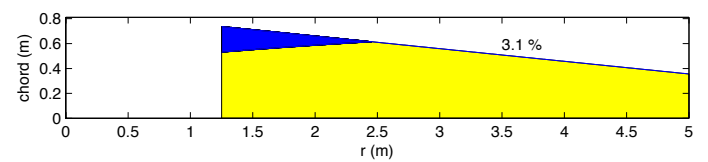

b)

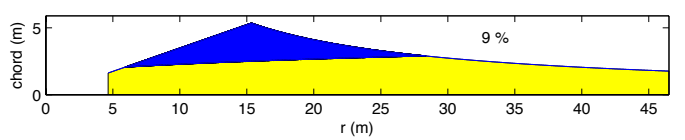

c)

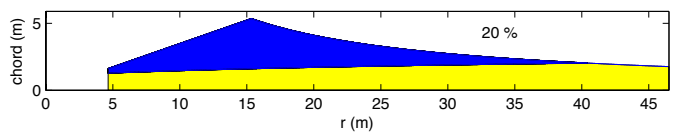

Fig. 7. Application of the criterion for rotational augmentation to a) the NREL turbine $[3,5]$ at a wind speed of $10 \mathrm{~m} / \mathrm{s}$. b) a $2.3 \mathrm{MW}$ turbine at a wind speed of $4 \mathrm{~m} / \mathrm{s}$, and c) the $2.3 \mathrm{MW}$ turbine at $13 \mathrm{~m} / \mathrm{s}$.

\section{Conclusion}

A systematic study of the developing laminar boundary layer over a rotating surface has succeeded in reproducing the key flow characteristics associated with rotational augmentation observed in wind turbines. The simulations suggested that the effect of rotation becomes important when the Ekman layer becomes thinner than the nonrotating developing boundary layer. At that point, the boundary layer stops growing, radially outwards flow is set, and vertical flow downwards from the free stream into the boundary layer is induced.

A criterion for the possibility of rotational augmentation was formulated and transferred to the more realistic case of a turbulent boundary layer. Applying the criterion to published evidence for rotational augmentation showed qualitative agreement with the literature. This suggests that the criterion, and the processes invoked in proposing it, have correctly identified the roles which the Ekman layer and centrifugal forces play in rotational augmentation.

\section{References}

[1] H. Himmelskamp (1947), Profile investigations on a rotating airscrew. Technical Report 832.

[2] H. Dwyer and W. McCroskey (1971), "Crossflow and unsteady boundary-layer effects on rotating blades", in AIAA Journal, Vol. 8, pp. 1498 - 1505.

[3] S. Schreck and M. Robinson (2004), "Tip speed ratio influences on rotationally augmented boundary layer topology and aerodynamic force generation" in Trans ASME - J. Solar Energy Eng., Vol. 126, pp. 1025-1033.

[4] S.P. Breton, F.N. Coton and G. Moe (2008), "A study on rotational effects and different stall delay models using a prescribed wake vortex scheme and NREL Phase VI experiment data", in Wind Energy, Vol. 11, pp. 459-482.

[5] S. Schreck, N. Sørensen and M. Robinson (2007), "Aerodynamic structures and processes in rotationally augmented flow fields", in Wind Energy, Vol. 10, pp. $159-178$.

[6] W. Banks and G. Gadd (1963) "Delaying effect of rotation on laminar separation", in AIAA Journal, Vol.1, pp.941-2

[7] H. Snel, R. Houwink and J. Bosschers (1994), Sectional prediction of lift coefficients on rotating wind turbine blades in stall. Technical Report ECN-C- 93-052. Netherlands Energy Research Foundation.

[8] Z. Du and M. Selig (2000), "The effect of rotation on the boundary layer of a wind turbine blade", in Renewable Energy Vol. 20, pp. $167-181$.

[9] E.M. Smirnov and A.V. Shatrov (1982), "Development of the boundary layer on a plate in a rotating system", in Fluid Dynamics, Vol. 17, pp. $452-455$.

[10] J. Pedlosky (1987), Geophysical Fluid Dynamics. SpringerVerlag, Berlin, Heidelberg, New York. second edition.

[11] B. Massey and J. Ward-Smith (1998), Mechanics of Fluids. Stanley Thornes, seventh edition.

[12] H.A. Khaledi, M. Barri and H.I. Andersson (2009), "On the stabilizing effect of the Coriolis force on the turbulent wake of a normal flat plate", in Phys. of Fluids, Vol. 21, 095104.

[13] K. Sakamoto and K. Akitomo (2008), "The tidally induced bottom boundary layer in a rotating frame: similarity of turbulence", in Journal of Fluid Mechanics, Vol.615, pp. $1-25$.

[14] I. Esau (2012), "Large scale turbulence structures in the Ekman boundary layer", in Geofizika, Vol. 29, pp. 5-34. 